Acta Crystallographica Section E

Structure Reports

Online

ISSN 1600-5368

\section{(E)-5-Phenyl-N-(2-thienylmethylene)- 1,3,4-thiadiazole-2-amine}

\section{Güneș Demirtaș, ${ }^{a}$ Necmi Dege, ${ }^{a}$ Memet Șekerci, ${ }^{b}$ Süleyman Servi ${ }^{\mathrm{b}}$ and Muharrem Dinçer ${ }^{\mathrm{a} *}$}

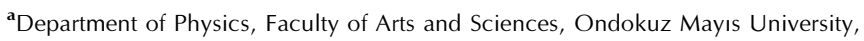
55139 Samsun, Turkey, and ${ }^{\mathbf{b}}$ Department of Chemistry, Faculty of Arts and Sciences, Fırat Universty, 38039 Elazığ, Turkey

Correspondence e-mail: gunesd@omu.edu.tr

Received 16 June 2009; accepted 18 June 2009

Key indicators: single-crystal X-ray study; $T=293 \mathrm{~K}$; mean $\sigma(\mathrm{C}-\mathrm{C})=0.002 \AA$; $R$ factor $=0.026 ; w R$ factor $=0.080 ;$ data-to-parameter ratio $=14.9$.

In the title compound, $\mathrm{C}_{13} \mathrm{H}_{9} \mathrm{~N}_{3} \mathrm{~S}_{2}$, the thiophene and phenyl rings are oriented at dihedral angles of $8.00(7)$ and $6.31(7)^{\circ}$, respectively, with respect to the central thiadiazole ring. No significant $\mathrm{C}-\mathrm{H} \cdots \mathrm{S}$ and $\pi-\pi$ interactions exist in the crystal structure.

\section{Related literature}

For the biological activity of $[1,3,4]$-thiadiazole-containing compounds, see: Foroumadi, Soltani et al. (2003); Foroumadi, Mansouri et al. (2003); Holla et al. (2002); Genc \& Servi (2005); Servi et al. (2005). For a related structure, see: Dege et al. (2006).

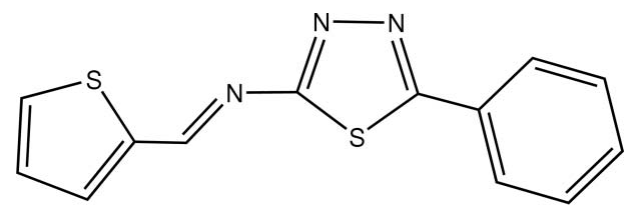

\section{Experimental}

Crystal data

$\mathrm{C}_{13} \mathrm{H}_{9} \mathrm{~N}_{3} \mathrm{~S}_{2} \quad M_{r}=271.35$

$$
\begin{aligned}
& \text { Monoclinic, } P 2_{1} / c \\
& a=6.2238(3) \AA \\
& b=7.7393(3) \AA \\
& c=25.6959(13) \AA \\
& \beta=94.701(4)^{\circ} \\
& V=1233.55(10) \AA^{3}
\end{aligned}
$$

Data collection

Stoe IPDS-2 diffractometer

Absorption correction: integration ( $X$-RED; Stoe \& Cie, 2002)

$T_{\min }=0.815, T_{\max }=0.943$

$Z=4$

Mo $K \alpha$ radiation

$\mu=0.41 \mathrm{~mm}^{-1}$

$T=293 \mathrm{~K}$

$0.74 \times 0.48 \times 0.16 \mathrm{~mm}$

Refinement

$R\left[F^{2}>2 \sigma\left(F^{2}\right)\right]=0.026$

$w R\left(F^{2}\right)=0.080$

$S=1.05$

2619 reflections

176 parameters
10930 measured reflections 2619 independent reflections 2275 reflections with $I>2 \sigma(I)$

$R_{\text {int }}=0.020$

Data collection: $X$-AREA (Stoe \& Cie, 2002); cell refinement: $X$ $A R E A$; data reduction: $X-R E D$ (Stoe \& Cie, 2002); program(s) used to solve structure: SHELXS97 (Sheldrick, 2008); program(s) used to refine structure: SHELXL97 (Sheldrick, 2008); molecular graphics: ORTEP-3 for Windows (Farrugia, 1997); software used to prepare material for publication: WinGX (Farrugia, 1999) and PLATON (Spek, 2009).

The authors thank the Ondokuz Mayis University Research Fund for financial support for this study.

Supplementary data and figures for this paper are available from the IUCr electronic archives (Reference: CI2829).

\title{
References
}

Dege, N. M., Şekerci, M. S., Servi, S. M., Dinçer, M. Ü. \& Demirbaş, Ü. (2006). Turk. J. Chem. 30, 103-108.

Farrugia, L. J. (1997). J. Appl. Cryst. 30, 565.

Farrugia, L. J. (1999). J. Appl. Cryst. 32, 837-838.

Foroumadi, A., Mansouri, S., Kiani, Z. \& Rahmani, A. (2003). Eur. J. Med. Chem. 38, 851-854.

Foroumadi, A., Soltani, F., Moshafi, M. H. \& Ashraf-Askari, R. (2003). Farmaco, 58, 1023-1028.

Genc, M. \& Servi, S. (2005). Heteroat. Chem. 16, 142-147.

Holla, B. S., Poorjary, K. N., Rao, B. S. \& Shivananda, M. K. (2002). Eur. J. Med. Chem. 37, 511-517.

Servi, S., Genc, M., Gur, S. \& Koca, M. (2005). Eur. J. Med. Chem. 40, 687-693. Sheldrick, G. M. (2008). Acta Cryst. A64, 112-122.

Spek, A. L. (2009). Acta Cryst. D65, 148-155.

Stoe \& Cie (2002). $X$-AREA and $X$-RED32. Stoe \& Cie, Darmstadt, Germany. 


\title{
supporting information
}

Acta Cryst. (2009). E65, o1668 [doi:10.1107/S1600536809023447]

\section{(E)-5-Phenyl-N-(2-thienylmethylene)-1,3,4-thiadiazole-2-amine}

\author{
Güneş Demirtaş, Necmi Dege, Memet Şekerci, Süleyman Servi and Muharrem Dinçer
}

\section{S1. Comment}

$[1,3,4]$-Thiadiazoles and their derivatives exhibit diverse biological activites possibly due to the presence of $=\mathrm{N}-\mathrm{C}-\mathrm{S}$ moiety (Holla et al., 2002; Servi et al., 2005; Genc \& Servi, 2005). Various phenyl substituted [1,3,4]-thiazole-2-amines and their derivatives have recently received significant importance because of their diverse biological properties (Foroumadi, Soltani et al., 2003; Foroumadi, Mansouri et al., 2003).We report here the crystal structure of the title compound, (I).

In (I), the C7-S1 [1.7234 (13) $\AA]$ distance is shorter than the C8-S1 distance [1.7411 (13) $\AA]$. The C11-S2 distance of 1.6993 (17) $\AA$ is shorter the $\mathrm{C} 10-\mathrm{S} 2$ distance of 1.7229 (13) $\AA$ and other $\mathrm{C}-\mathrm{S}$ bonds in the molecule. These bond distances agree well with the corresponding values reported for 1-(thiophen-2-ylmethyl)-2-(thiophen-2-yl)-1Hbenzimidazole (Dege et al., 2006). The thiophene and phenyl rings are oriented at dihedral angles of $8.00(7)^{\circ}$ and $6.31(7)^{\circ}$, respectively, with respect to the central thiadiazole ring.

No significant $\mathrm{C}-\mathrm{H} \cdots \mathrm{S}$ and $\pi-\pi$ interactions are observed.

\section{S2. Experimental}

A solution of 5-phenyl-1,3,4-thiadiazole-2-amine $(0.01 \mathrm{~mol})$ in absolute ethanol $(20 \mathrm{ml})$ was added in small portions to a solution of thiophen-2-carbaldehyde $(0.01 \mathrm{~mol})$ in absolute ethanol $(30 \mathrm{ml})$. The reaction mixture was maintained at 343 $\mathrm{K}$ for $4 \mathrm{~h}$, cooled and then added to ice-cold water. The resulting solid was washed with water, dried and recrystallized from ethanol (yield 77\%). IR $\left(\mathrm{cm}^{-1}\right)$ : $3078(\mathrm{Ar} \mathrm{H}), 1633(\mathrm{C}=\mathrm{C}), 1589(\mathrm{C}=\mathrm{N}) ;{ }^{1} \mathrm{H}-\mathrm{NMR}$ : 7.2-7.9 (m, 8H, aromatic protons), $9.2\left(\mathrm{~s},{ }^{1} \mathrm{H}, \mathrm{N}=\mathrm{CH}\right.$ proton).

\section{S3. Refinement}

Atoms H1, H9 and $\mathrm{H} 12$ were located in a difference map and refined freely. The other $\mathrm{H}$ atoms were positioned geometrically and refined using a riding model, with $\mathrm{C}-\mathrm{H}=0.93 \AA$ and $U_{\mathrm{iso}}(\mathrm{H})=1.2 \mathrm{U}_{\mathrm{eq}}(\mathrm{C})$. 


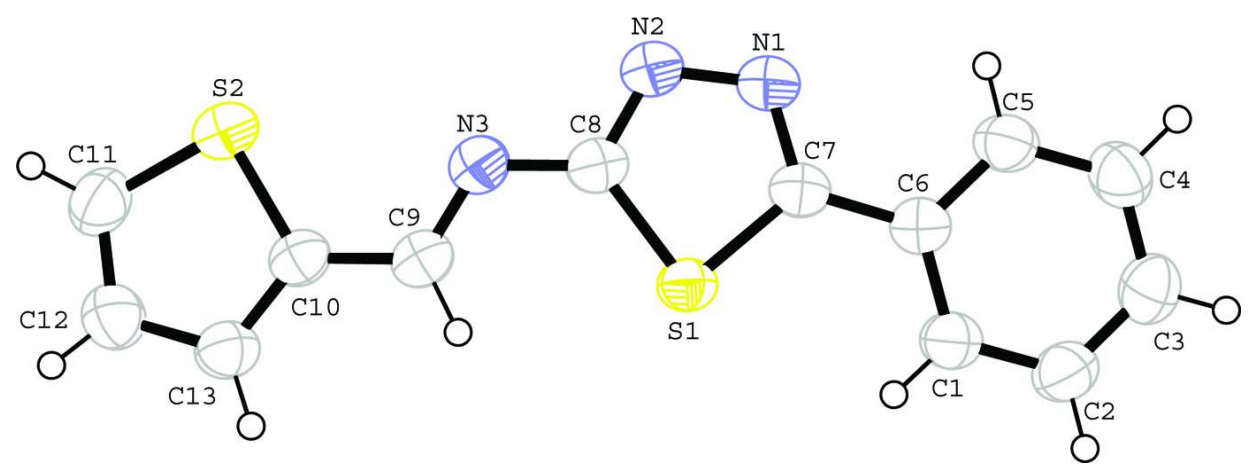

\section{Figure 1}

The molecular structure of (I), with the atom-numbering scheme. Displacement ellipsoids are drawn at the 50\% probability level.

\section{(E)-5-Phenyl-N-(2-thienylmethylene)-1,3,4-thiadiazole-2-amine}

\section{Crystal data}

$\mathrm{C}_{13} \mathrm{H}_{9} \mathrm{~N}_{3} \mathrm{~S}_{2}$

$M_{r}=271.35$

Monoclinic, $P 2_{1} / c$

Hall symbol: -P $2 \mathrm{ybc}$

$a=6.2238(3) \AA$

$b=7.7393(3) \AA$

$c=25.6959(13) \AA$

$\beta=94.701(4)^{\circ}$

$V=1233.55(10) \AA^{3}$

$Z=4$

\section{Data collection}

Stoe IPDS-2

diffractometer

Radiation source: fine-focus sealed tube

Plane graphite monochromator

Detector resolution: 6.67 pixels $\mathrm{mm}^{-1}$

$\omega$ scans

Absorption correction: integration

(X-RED; Stoe \& Cie, 2002)

$T_{\min }=0.815, T_{\max }=0.943$

\section{Refinement}

Refinement on $F^{2}$

Least-squares matrix: full

$R\left[F^{2}>2 \sigma\left(F^{2}\right)\right]=0.026$

$w R\left(F^{2}\right)=0.080$

$S=1.05$

2619 reflections

176 parameters

0 restraints

Primary atom site location: structure-invariant direct methods

Secondary atom site location: difference Fourier map
$F(000)=560$

$D_{\mathrm{x}}=1.461 \mathrm{Mg} \mathrm{m}^{-3}$

Mo $K \alpha$ radiation, $\lambda=0.71073 \AA$

Cell parameters from 17067 reflections

$\theta=1.6-26.8^{\circ}$

$\mu=0.41 \mathrm{~mm}^{-1}$

$T=293 \mathrm{~K}$

Plate, yellow

$0.74 \times 0.48 \times 0.16 \mathrm{~mm}$

10930 measured reflections

2619 independent reflections

2275 reflections with $I>2 \sigma(I)$

$R_{\text {int }}=0.020$

$\theta_{\text {max }}=26.8^{\circ}, \theta_{\min }=1.6^{\circ}$

$h=-7 \rightarrow 7$

$k=-9 \rightarrow 9$

$l=-32 \rightarrow 32$

Hydrogen site location: inferred from neighbouring sites

$\mathrm{H}$ atoms treated by a mixture of independent and constrained refinement

$w=1 /\left[\sigma^{2}\left(F_{\mathrm{o}}^{2}\right)+(0.0481 P)^{2}+0.1148 P\right]$

where $P=\left(F_{\mathrm{o}}{ }^{2}+2 F_{\mathrm{c}}{ }^{2}\right) / 3$

$(\Delta / \sigma)_{\max }=0.001$

$\Delta \rho_{\max }=0.18$ e $\AA^{-3}$

$\Delta \rho_{\min }=-0.17$ e $\AA^{-3}$

Extinction correction: SHELXL97 (Sheldrick, 2008), $\mathrm{Fc}^{*}=\mathrm{kFc}\left[1+0.001 \mathrm{xFc}^{2} \lambda^{3} / \sin (2 \theta)\right]^{-1 / 4}$

Extinction coefficient: 0.002634 (11) 


\section{Special details}

Geometry. All e.s.d.'s (except the e.s.d. in the dihedral angle between two 1.s. planes) are estimated using the full covariance matrix. The cell e.s.d.'s are taken into account individually in the estimation of e.s.d.'s in distances, angles and torsion angles; correlations between e.s.d.'s in cell parameters are only used when they are defined by crystal symmetry. An approximate (isotropic) treatment of cell e.s.d.'s is used for estimating e.s.d.'s involving 1.s. planes.

Refinement. Refinement of $F^{2}$ against ALL reflections. The weighted $R$-factor $w R$ and goodness of fit $S$ are based on $F^{2}$, conventional $R$-factors $R$ are based on $F$, with $F$ set to zero for negative $F^{2}$. The threshold expression of $F^{2}>\sigma\left(F^{2}\right)$ is used only for calculating $R$-factors (gt) etc. and is not relevant to the choice of reflections for refinement. $R$-factors based on $F^{2}$ are statistically about twice as large as those based on $F$, and $R$ - factors based on ALL data will be even larger.

Fractional atomic coordinates and isotropic or equivalent isotropic displacement parameters $\left(\AA^{2}\right)$

\begin{tabular}{lllll}
\hline & $x$ & $y$ & $z$ & $U_{\text {iso }} / U_{\text {eq }}$ \\
\hline C1 & $0.0290(2)$ & $0.1603(2)$ & $0.57838(6)$ & $0.0543(3)$ \\
C2 & $0.0105(3)$ & $0.0893(2)$ & $0.62710(6)$ & $0.0615(4)$ \\
H2 & 0.1311 & 0.0817 & 0.6509 & $0.074^{*}$ \\
C3 & $-0.1859(3)$ & $0.0297(2)$ & $0.64059(6)$ & $0.0621(4)$ \\
H3 & -0.1976 & -0.0190 & 0.6733 & $0.075^{*}$ \\
C4 & $-0.3643(2)$ & $0.0422(2)$ & $0.60563(6)$ & $0.0627(4)$ \\
H4 & -0.4967 & 0.0014 & 0.6147 & $0.075^{*}$ \\
C5 & $-0.3479(2)$ & $0.1152(2)$ & $0.55709(6)$ & $0.0565(3)$ \\
H5 & -0.4698 & 0.1247 & 0.5338 & $0.068^{*}$ \\
C6 & $-0.1503(2)$ & $0.17452(16)$ & $0.54281(5)$ & $0.0461(3)$ \\
C7 & $-0.13539(19)$ & $0.24791(17)$ & $0.49053(5)$ & $0.0466(3)$ \\
C8 & $-0.0473(2)$ & $0.36506(18)$ & $0.40911(5)$ & $0.0500(3)$ \\
C9 & $0.2276(2)$ & $0.46866(18)$ & $0.36247(5)$ & $0.0500(3)$ \\
C10 & $0.3030(2)$ & $0.55163(17)$ & $0.31753(5)$ & $0.0480(3)$ \\
C11 & $0.3279(3)$ & $0.7018(2)$ & $0.23422(6)$ & $0.0643(4)$ \\
H11 & 0.3078 & 0.7541 & 0.2016 & $0.077^{*}$ \\
C12 & $0.5205(3)$ & $0.6920(2)$ & $0.26256(7)$ & $0.0631(4)$ \\
C13 & $0.5080(2)$ & $0.60554(18)$ & $0.31021(6)$ & $0.0547(3)$ \\
H13 & 0.6259 & 0.5869 & 0.3342 & $0.066^{*}$ \\
N1 & $-0.30366(19)$ & $0.27346(19)$ & $0.45777(5)$ & $0.0656(4)$ \\
N2 & $-0.25288(19)$ & $0.3405(2)$ & $0.41099(5)$ & $0.0674(4)$ \\
N3 & $0.02776(18)$ & $0.43483(15)$ & $0.36467(4)$ & $0.0530(3)$ \\
S1 & $0.10458(5)$ & $0.30465(5)$ & $0.466131(13)$ & $0.04910(12)$ \\
S2 & $0.12673(6)$ & $0.60757(5)$ & $0.265024(14)$ & $0.06061(14)$ \\
H9 & $0.333(3)$ & $0.444(2)$ & $0.3905(6)$ & $0.065(4)^{*}$ \\
H1 & $0.158(3)$ & $0.206(2)$ & $0.5694(7)$ & $0.071(5)^{*}$ \\
H12 & $0.645(3)$ & $0.736(3)$ & $0.2497(8)$ & $0.086(6)^{*}$ \\
& & & &
\end{tabular}

Atomic displacement parameters $\left(\AA^{2}\right)$

\begin{tabular}{lllllll}
\hline & $U^{11}$ & $U^{22}$ & $U^{33}$ & $U^{12}$ & $U^{13}$ & $U^{23}$ \\
\hline C1 & $0.0472(7)$ & $0.0616(8)$ & $0.0537(7)$ & $-0.0008(6)$ & $0.0013(6)$ & $-0.0007(6)$ \\
C2 & $0.0579(8)$ & $0.0733(10)$ & $0.0520(8)$ & $0.0033(7)$ & $-0.0038(6)$ & $0.0016(7)$ \\
C3 & $0.0714(9)$ & $0.0656(9)$ & $0.0502(8)$ & $0.0040(7)$ & $0.0103(7)$ & $0.0006(7)$ \\
C4 & $0.0557(8)$ & $0.0725(10)$ & $0.0617(9)$ & $-0.0027(7)$ & $0.0151(7)$ & $-0.0015(7)$ \\
C5 & $0.0455(7)$ & $0.0690(9)$ & $0.0549(8)$ & $0.0005(6)$ & $0.0033(6)$ & $-0.0047(7)$
\end{tabular}


supporting information

$\begin{array}{lllllll}\text { C6 } & 0.0460(6) & 0.0435(7) & 0.0486(6) & 0.0036(5) & 0.0032(5) & -0.0061(5) \\ \text { C7 } & 0.0425(6) & 0.0452(6) & 0.0516(7) & 0.0016(5) & 0.0007(5) & -0.0035(5) \\ \text { C8 } & 0.0466(7) & 0.0514(7) & 0.0509(7) & 0.0011(5) & -0.0026(5) & 0.0001(6) \\ \text { C9 } & 0.0513(7) & 0.0494(7) & 0.0483(7) & 0.0046(6) & -0.0021(5) & -0.0003(6) \\ \text { C10 } & 0.0496(7) & 0.0469(7) & 0.0467(7) & 0.0051(5) & -0.0010(5) & -0.0019(5) \\ \text { C11 } & 0.0729(10) & 0.0655(9) & 0.0537(8) & -0.0013(7) & 0.0004(7) & 0.0108(7) \\ \text { C12 } & 0.0590(8) & 0.0636(10) & 0.0670(9) & -0.0037(7) & 0.0073(7) & 0.0085(7) \\ \text { C13 } & 0.0503(7) & 0.0552(8) & 0.0576(8) & 0.0031(6) & -0.0020(6) & 0.0031(6) \\ \text { N1 } & 0.0443(6) & 0.0864(9) & 0.0647(8) & -0.0049(6) & -0.0039(5) & 0.0167(7) \\ \text { N2 } & 0.0479(6) & 0.0901(10) & 0.0622(7) & -0.0051(6) & -0.0068(5) & 0.0208(7) \\ \text { N3 } & 0.0518(6) & 0.0564(7) & 0.0499(6) & -0.0003(5) & -0.0009(5) & 0.0045(5) \\ \text { S1 } & 0.04071(18) & 0.0581(2) & 0.04783(19) & 0.00331(13) & -0.00016(12) & 0.00054(14) \\ \text { S2 } & 0.0538(2) & 0.0724(3) & 0.0536(2) & -0.00062(16) & -0.00781(15) & 0.00514(17) \\ & & & & & \end{array}$

Geometric parameters $\left(\AA,{ }^{\circ}\right)$

\begin{tabular}{|c|c|c|c|}
\hline $\mathrm{C} 1-\mathrm{C} 2$ & $1.381(2)$ & $\mathrm{C} 8-\mathrm{N} 3$ & $1.3787(18)$ \\
\hline $\mathrm{C} 1-\mathrm{C} 6$ & $1.3875(19)$ & $\mathrm{C} 8-\mathrm{S} 1$ & $1.7411(13)$ \\
\hline $\mathrm{C} 1-\mathrm{H} 1$ & $0.923(18)$ & $\mathrm{C} 9-\mathrm{N} 3$ & $1.2770(18)$ \\
\hline $\mathrm{C} 2-\mathrm{C} 3$ & $1.377(2)$ & $\mathrm{C} 9-\mathrm{C} 10$ & 1.4335 (19) \\
\hline $\mathrm{C} 2-\mathrm{H} 2$ & 0.93 & C9-H9 & $0.952(16)$ \\
\hline $\mathrm{C} 3-\mathrm{C} 4$ & $1.374(2)$ & $\mathrm{C} 10-\mathrm{C} 13$ & $1.3697(19)$ \\
\hline $\mathrm{C} 3-\mathrm{H} 3$ & 0.93 & $\mathrm{C} 10-\mathrm{S} 2$ & $1.7229(13)$ \\
\hline $\mathrm{C} 4-\mathrm{C} 5$ & $1.380(2)$ & $\mathrm{C} 11-\mathrm{C} 12$ & $1.353(2)$ \\
\hline $\mathrm{C} 4-\mathrm{H} 4$ & 0.93 & $\mathrm{C} 11-\mathrm{S} 2$ & $1.6993(17)$ \\
\hline $\mathrm{C} 5-\mathrm{C} 6$ & $1.3900(19)$ & C11-H11 & 0.93 \\
\hline $\mathrm{C} 5-\mathrm{H} 5$ & 0.93 & $\mathrm{C} 12-\mathrm{C} 13$ & $1.403(2)$ \\
\hline $\mathrm{C} 6-\mathrm{C} 7$ & $1.4684(19)$ & $\mathrm{C} 12-\mathrm{H} 12$ & 0.929 (19) \\
\hline $\mathrm{C} 7-\mathrm{N} 1$ & $1.3039(17)$ & $\mathrm{C} 13-\mathrm{H} 13$ & 0.93 \\
\hline $\mathrm{C} 7-\mathrm{S} 1$ & $1.7234(13)$ & $\mathrm{N} 1-\mathrm{N} 2$ & $1.3696(19)$ \\
\hline $\mathrm{C} 8-\mathrm{N} 2$ & $1.2987(18)$ & & \\
\hline $\mathrm{C} 2-\mathrm{C} 1-\mathrm{C} 6$ & $120.44(14)$ & $\mathrm{N} 2-\mathrm{C} 8-\mathrm{S} 1$ & $113.44(11)$ \\
\hline $\mathrm{C} 2-\mathrm{C} 1-\mathrm{H} 1$ & $121.1(12)$ & $\mathrm{N} 3-\mathrm{C} 8-\mathrm{S} 1$ & $127.22(10)$ \\
\hline $\mathrm{C} 6-\mathrm{C} 1-\mathrm{H} 1$ & $118.3(11)$ & $\mathrm{N} 3-\mathrm{C} 9-\mathrm{C} 10$ & $120.78(12)$ \\
\hline $\mathrm{C} 3-\mathrm{C} 2-\mathrm{C} 1$ & $120.20(14)$ & N3-C9-H9 & $122.7(10)$ \\
\hline $\mathrm{C} 3-\mathrm{C} 2-\mathrm{H} 2$ & 119.9 & $\mathrm{C} 10-\mathrm{C} 9-\mathrm{H} 9$ & $116.5(10)$ \\
\hline $\mathrm{C} 1-\mathrm{C} 2-\mathrm{H} 2$ & 119.9 & $\mathrm{C} 13-\mathrm{C} 10-\mathrm{C} 9$ & $128.09(12)$ \\
\hline $\mathrm{C} 4-\mathrm{C} 3-\mathrm{C} 2$ & $119.90(14)$ & $\mathrm{C} 13-\mathrm{C} 10-\mathrm{S} 2$ & $110.88(10)$ \\
\hline $\mathrm{C} 4-\mathrm{C} 3-\mathrm{H} 3$ & 120.0 & $\mathrm{C} 9-\mathrm{C} 10-\mathrm{S} 2$ & $120.94(10)$ \\
\hline $\mathrm{C} 2-\mathrm{C} 3-\mathrm{H} 3$ & 120.0 & $\mathrm{C} 12-\mathrm{C} 11-\mathrm{S} 2$ & $112.30(12)$ \\
\hline $\mathrm{C} 3-\mathrm{C} 4-\mathrm{C} 5$ & $120.28(14)$ & $\mathrm{C} 12-\mathrm{C} 11-\mathrm{H} 11$ & 123.9 \\
\hline $\mathrm{C} 3-\mathrm{C} 4-\mathrm{H} 4$ & 119.9 & $\mathrm{~S} 2-\mathrm{C} 11-\mathrm{H} 11$ & 123.9 \\
\hline $\mathrm{C} 5-\mathrm{C} 4-\mathrm{H} 4$ & 119.9 & $\mathrm{C} 11-\mathrm{C} 12-\mathrm{C} 13$ & $112.73(14)$ \\
\hline $\mathrm{C} 4-\mathrm{C} 5-\mathrm{C} 6$ & $120.40(14)$ & $\mathrm{C} 11-\mathrm{C} 12-\mathrm{H} 12$ & $121.0(13)$ \\
\hline $\mathrm{C} 4-\mathrm{C} 5-\mathrm{H} 5$ & 119.8 & $\mathrm{C} 13-\mathrm{C} 12-\mathrm{H} 12$ & $126.3(13)$ \\
\hline $\mathrm{C} 6-\mathrm{C} 5-\mathrm{H} 5$ & 119.8 & $\mathrm{C} 10-\mathrm{C} 13-\mathrm{C} 12$ & $112.57(13)$ \\
\hline $\mathrm{C} 1-\mathrm{C} 6-\mathrm{C} 5$ & $118.77(13)$ & $\mathrm{C} 10-\mathrm{C} 13-\mathrm{H} 13$ & 123.7 \\
\hline
\end{tabular}




$\begin{array}{llll}\mathrm{C} 1-\mathrm{C} 6-\mathrm{C} 7 & 121.70(12) & \mathrm{C} 12-\mathrm{C} 13-\mathrm{H} 13 & 123.7 \\ \mathrm{C} 5-\mathrm{C} 6-\mathrm{C} 7 & 119.53(12) & \mathrm{C} 7-\mathrm{N} 1-\mathrm{N} 2 & 113.15(12) \\ \mathrm{N} 1-\mathrm{C} 7-\mathrm{C} 6 & 122.79(12) & \mathrm{C} 8-\mathrm{N} 2-\mathrm{N} 1 & 112.78(12) \\ \mathrm{N} 1-\mathrm{C} 7-\mathrm{S} 1 & 113.62(11) & \mathrm{C} 9-\mathrm{N} 3-\mathrm{C} 8 & 120.94(12) \\ \mathrm{C} 6-\mathrm{C} 7-\mathrm{S} 1 & 123.58(9) & \mathrm{C} 7-\mathrm{S} 1-\mathrm{C} 8 & 87.01(6) \\ \mathrm{N} 2-\mathrm{C} 8-\mathrm{N} 3 & 119.33(12) & \mathrm{C} 11-\mathrm{S} 2-\mathrm{C} 10 & 91.51(7) \\ & & & -0.5(2) \\ \mathrm{C} 6-\mathrm{C} 1-\mathrm{C} 2-\mathrm{C} 3 & 0.9(2) & \mathrm{C} 11-\mathrm{C} 12-\mathrm{C} 13-\mathrm{C} 10 & -179.04(13) \\ \mathrm{C} 1-\mathrm{C} 2-\mathrm{C} 3-\mathrm{C} 4 & -0.5(2) & \mathrm{C} 6-\mathrm{C} 7-\mathrm{N} 1-\mathrm{N} 2 & -0.15(18) \\ \mathrm{C} 2-\mathrm{C} 3-\mathrm{C} 4-\mathrm{C} 5 & -0.3(2) & \mathrm{S} 1-\mathrm{C} 7-\mathrm{N} 1-\mathrm{N} 2 & -178.89(13) \\ \mathrm{C} 3-\mathrm{C} 4-\mathrm{C} 5-\mathrm{C} 6 & 0.9(2) & \mathrm{N} 3-\mathrm{C} 8-\mathrm{N} 2-\mathrm{N} 1 & 0.45(19) \\ \mathrm{C} 2-\mathrm{C} 1-\mathrm{C} 6-\mathrm{C} 5 & -0.3(2) & \mathrm{S} 1-\mathrm{C} 8-\mathrm{N} 2-\mathrm{N} 1 & -0.2(2) \\ \mathrm{C} 2-\mathrm{C} 1-\mathrm{C} 6-\mathrm{C} 7 & -179.40(13) & \mathrm{C} 7-\mathrm{N} 1-\mathrm{N} 2-\mathrm{C} 8 & -175.86(12) \\ \mathrm{C} 4-\mathrm{C} 5-\mathrm{C} 6-\mathrm{C} 1 & -0.6(2) & \mathrm{C} 10-\mathrm{C} 9-\mathrm{N} 3-\mathrm{C} 8 & -2.50(15) \\ \mathrm{C} 4-\mathrm{C} 5-\mathrm{C} 6-\mathrm{C} 7 & 178.55(14) & \mathrm{N} 2-\mathrm{C} 8-\mathrm{N} 3-\mathrm{C} 9 & 0.32(12) \\ \mathrm{C} 1-\mathrm{C} 6-\mathrm{C} 7-\mathrm{N} 1 & -175.17(14) & \mathrm{S} 1-\mathrm{C} 8-\mathrm{N} 3-\mathrm{C} 9 & 179.20(12) \\ \mathrm{C} 5-\mathrm{C} 6-\mathrm{C} 7-\mathrm{N} 1 & 5.7(2) & \mathrm{N} 1-\mathrm{C} 7-\mathrm{S} 1-\mathrm{C} 8 & -0.44(13) \\ \mathrm{C} 1-\mathrm{C} 6-\mathrm{C} 7-\mathrm{S} 1 & 6.05(19) & \mathrm{C} 6-\mathrm{C} 7-\mathrm{S} 1-\mathrm{C} 8 & 178.84(13) \\ \mathrm{C} 5-\mathrm{C} 6-\mathrm{C} 7-\mathrm{S} 1 & -173.02(11) & \mathrm{N} 2-\mathrm{C} 8-\mathrm{S} 1-\mathrm{C} 7 & -0.24(14) \\ \mathrm{N} 3-\mathrm{C} 9-\mathrm{C} 10-\mathrm{C} 13 & 175.89(14) & \mathrm{N} 3-\mathrm{C} 8-\mathrm{S} 1-\mathrm{C} 7 & -0.04(12) \\ \mathrm{N} 3-\mathrm{C} 9-\mathrm{C} 10-\mathrm{S} 2 & -0.24(19) & \mathrm{C} 12-\mathrm{C} 11-\mathrm{S} 2-\mathrm{C} 10 & 176.70(12) \\ \mathrm{S} 2-\mathrm{C} 11-\mathrm{C} 12-\mathrm{C} 13 & 0.5(2) & \mathrm{C} 13-\mathrm{C} 10-\mathrm{S} 2-\mathrm{C} 11 & \\ \mathrm{C} 9-\mathrm{C} 10-\mathrm{C} 13-\mathrm{C} 12 & -176.14(14) & \mathrm{C} 9-\mathrm{C} 10-\mathrm{S} 2-\mathrm{C} 11 & \\ \mathrm{~S} 2-\mathrm{C} 10-\mathrm{C} 13-\mathrm{C} 12 & 0.30(17) & & \end{array}$

